\title{
Flutamide versus a cyproterone acetate-ethinyl estradiol combination in moderate acne: a pilot randomized clinical trial
}

This article was published in the following Dove Press journal:

Clinical, Cosmetic and Investigational Dermatology

22 July $201 \mathrm{I}$

Number of times this article has been viewed

\author{
Hassan Adalatkhah' \\ Farhad Pourfarzi ${ }^{2}$ \\ Homayoun \\ Sadeghi-Bazargani ${ }^{3}$ \\ 'Department of Dermatology, \\ ${ }^{2}$ Department of Social Medicine, \\ Faculty of Medicine, Ardabil \\ University of Medical Sciences, \\ Ardabil; ${ }^{3}$ Statistics and Epidemiology \\ Department, Faculty of Health \\ and Nutrition, Tabriz University \\ of Medical Sciences, Tabriz, Iran
}

Correspondence: Hassan Adalatkhah Department of Dermatology, Faculty of Medicine, Ardabil University of Medical Sciences, Raheh Daneshgah

Ave, Ardabil, Iran

Tel +989143086490

Fax +98 45I 77|48I8

Email h.edalatkhah@arums.ac.ir
Background: The use of oral flutamide is rarely investigated in acne therapy. The aim of this study was to compare the efficacy of oral flutamide with that of a cyproterone-estradiol combination in treating acne lesions.

Methods: A randomized clinical trial enrolled patients with moderate acne into two equal groups to receive either oral flutamide or the cyproterone-estradiol combination for 6 months. Lesion count, Acne Severity Index, and Global Acne Grading system (GAGS) scores were used to assess improvement in acne lesions. The dichotomous measurement scale for primary endpoint assessment was defined as improvement from moderate to mild acne based on GAGS score. Patient satisfaction and dermal fat were also assessed. Intention to treat and per protocol analyses were done, reporting related effect sizes.

Results: Both treatments resulted in substantial improvement in acne lesions. Although flutamide seemed to have higher efficacy, an intention to treat analysis did not find the two treatment protocols to be different. The relative risk in intention to treat analysis was 1.8 (95\% confidence interval [CI] 0.89-1.6), and was 1.33 (95\% CI 1.03-1.72) for the per protocol analysis. The number needed to treat for flutamide compared with the cyproterone-estradiol combination was 7.7 and 4.2 in the intention to treat and per protocol analyses, respectively.

Conclusion: Flutamide appears to be more effective than a cyproterone-estradiol combination in some aspects of acne treatment, but this requires confirmation in a larger trial.

Keywords: acne vulgaris, flutamide, cyproterone acetate, ethinyl estradiol, androgen antagonists

\section{Introduction}

Acne is attributed to many factors, including increased sebum production, altered quality of sebum lipids, regulation of cutaneous steroidogenesis, androgen activity, interaction with neuropeptides, proinflammatory and anti-inflammatory processes, follicular hyperkeratinization, and proliferation of Propionibacterium acnes within the follicle. ${ }^{1}$ A recent review of acne therapy encourages research into nonantibiotic therapies to minimize the risk of antibiotic resistance in the community. ${ }^{2}$ Recent research findings have highlighted the important role of hormones in the pathogenesis of acne. Androgens are believed to contribute to the development of acne, and this theory strengthens more when considering the interactions between sebum production, hirsutism, polycystic ovarian disease, and melasma with acne and androgens. ${ }^{3-7}$ Considering the pathophysiology of acne, newer steroid-related therapies need to be identified and investigated. Cyproterone and oral contraceptives are recommended for treatment of acne in the literature but are discouraged for long-term use. ${ }^{8,9}$ However, use of oral flutamide has rarely been 
investigated in acne therapy. Flutamide is a nonsteroidal peripheral androgen antagonist usually used to treat prostatic cancer. It lacks any progestogenic or antigonadotropic activity, and thus does not cause menstrual irregularity. ${ }^{10}$ The aim of this study was to compare the efficacy of oral flutamide with that of a cyproterone acetate-ethinyl estradiol combination in treating acne lesions.

\section{Methods}

This research was conducted in a specialty dermatology clinic during 2007-2008 as a randomized clinical trial with two parallel drug treatment arms. Females with moderate acne based on the Acne Severity Index (ASI) comprised the study population. The inclusion criteria were a complaint of moderate acne and age over 16 years. Exclusion criteria were pregnancy, lactation, hepatic disease, and use of oral contraceptives or androgenic drugs. Considering the paucity of information regarding the use of flutamide and its dosing, the study was conducted as a pilot investigation with a substantially small sample size, mainly due to ethical limitations and also due to practicability issues.

\section{Randomization and blinding}

Randomization lists were generated to allocate the subjects into two treatment groups. The evaluating physician was blinded to treatment type. A nurse coordinated the evaluation sessions, and was responsible for keeping the allocation codes, inviting the patients for each follow-up, coordinating physician visits, issuing blank evaluation forms, and collecting the forms to be filed. She also checked whether the patient had used the prescribed drug during each treatment period. The physician responsible for evaluation was asked to document whether unmasking had happened during evaluation or not. No unmasking occurred during the study.

\section{Treatment protocol}

The treatment regimen was continued for six months. The protocol in the first arm of the trial included flutamide $250 \mathrm{mg}$ daily (21 days in each month) and topical clindamycin 1\% for 6 months. The protocol in the second arm included a cyproterone compound containing $2 \mathrm{mg}$ cyproterone and $50 \mu \mathrm{g}$ ethinyl estradiol (21 days in each month) plus topical clindamycin $1 \%$ for 6 months.

\section{Outcome}

Improvement of acne lesions and greasy skin were assessed every 2 months using a Mexameter ${ }^{\circledR}$. The Mexameter determines an index for melanin and erythema, the main color giving the parameters of the skin very accurately. Lesion count, ASI, and Global Acne Grading system (GAGS) scores were used to assess improvement of acne lesions. ${ }^{11}$ The formula used to calculate ASI was $2 \times$ pustules $+1 \times$ papules + comedones $\times 25 \%$. Patient satisfaction regarding different aspects of acne treatment was also assessed.

\section{Statistical analysis}

Data were analyzed using the STATA 11 statistical software package (STATA Corporation, College Station, TX). Descriptive and bivariate statistical methods were used for background data analysis. Classical risk assessment methods used in clinical trials as well as regression methods were employed to compare drug efficacy regarding the primary endpoints. Statistical test results were assessed using a two-sided hypothesis testing strategy, and $P<0.05$ was considered to be statistically significant.

The GAGS score-based endpoint was defined on both continuous and dichotomous scales. Considering the repeated measurement nature of the data as well as missing values, a generalized estimation equation regression analysis was applied to assess outcome trends and adjust for possible confounders. ${ }^{12}$ The dichotomous measurement scale for primary endpoint assessment was defined as improvement from moderate to mild acne based on GAGS score. Relative risk, absolute risk difference, and number needed to treat statistics were calculated to compare the efficacy of flutamide with that of the cyproterone-estradiol combination. Continuous ASI and GAGS scores, as well as patient satisfaction, were considered as secondary endpoints.

Regarding the intention to treat analysis for missing outcomes, although generalized estimation equations can handle missing data reasonably, considering the strong assumption of the statistical methods when handling missing data, a logical outcome permutation method was used in the intention to treat analysis to compare drug efficacy after 6 months of treatment. Cases with fewer than two measurement occasions, a less than $50 \%$ decrease in lesion count (simultaneously for all three types of lesions), and cases with at least a $50 \%$ decrease in lesions but without a continuing decrement trend over the first three measurements, were considered as treatment failures at last measurement. Results of the intention to treat analysis were compared with those of the per protocol analysis. Nonadherers were eliminated from the per protocol analysis. The main conclusion about the efficacy of the investigational treatment was based on the intention to treat analysis. A flow diagram of enrolment and follow-up in the study is given in Figure 1. The study was approved by 


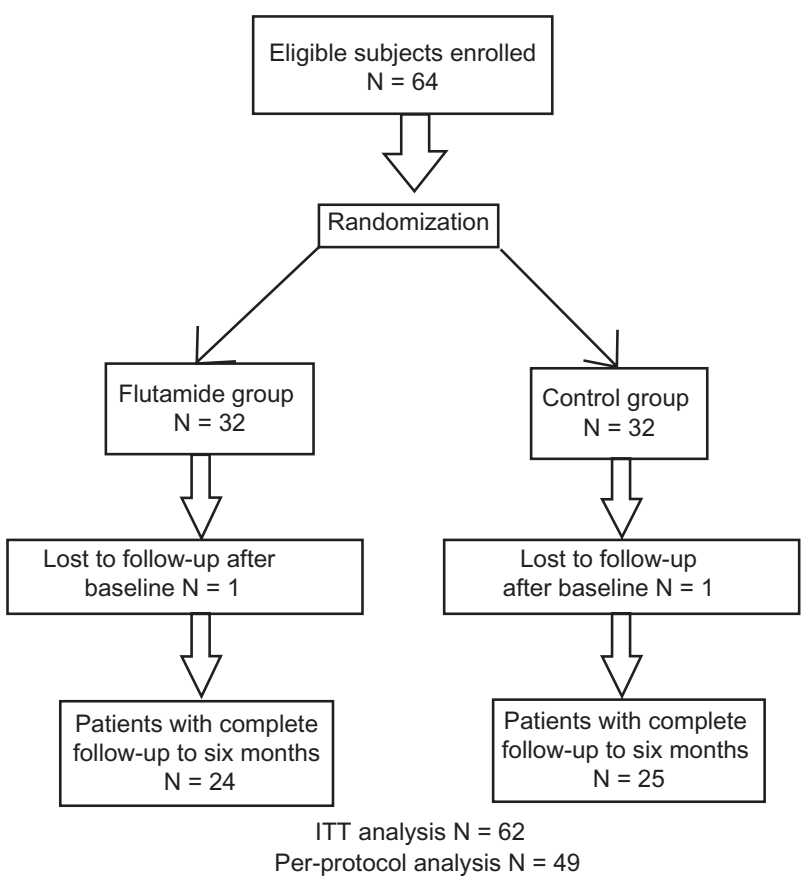

Figure I Flow diagram of trial enrolment and follow-up. Abbreviation: ITT, intention to treat.

the ethics committee of the Ardabil University of Medical Sciences. The study aim was explained to eligible patients and informed consent was obtained. The study was registered with a clinical trial registry center approved by the World Health Organization.

\section{Results}

One patient in each group did not attend the first follow-up after receiving their allocated treatment. Twenty-four patients in the flutamide group and 25 patients in the cyproteroneestradiol combination group completed the six-month follow-up period. Thirteen patients were lost to follow-up before 6 months (six from the cyproterone-estradiol combination group and seven from the flutamide group). Ten of these lost cases had improved from moderate acne to a mild or lower GAGS score during the first three months of treatment. One of the 13 patients in the cyproterone-estradiol combination group had aggravation of acne one month after treatment and stopped follow-up. Two patients from the flutamide group who had at least a $70 \%$ decrement in number of comedones, papules, and pustules one month after treatment also stopped follow-up.

The mean age of the patients was 22 years. The patients had had acne on average for 3 years before enrollment. Thirty-seven percent reported a previous history of ovarian cysts. Half of the patients had a positive family history of acne in their sisters, $40 \%$ in their brothers, and less than $10 \%$ in their parents. Fifty-seven percent had previously received acne treatment, more than $90 \%$ of whom had received both topical and oral treatments. Topical clindamycin had been used by $45 \%$ of patients before. One patient had used erythromycin and no patient mentioned using retinoic acid or benzoyl peroxide. No case of pregnancy or abortion occurred during this trial.

In a descriptive sense, as shown in Figure 2, the ASI and GAGS scores showed a trend of improvement in the flutamide group versus the cyproterone-estradiol combination group. The amount of sebum showed a decreasing trend overall (within-group $P<0.001$ ) and prominently for the flutamide group ( $\beta=-4.3$; between-group $P<0.01$ ). Comedone, papule, and pustule counts showed a decreasing trend overall and on each measurement occasion $(P<0.001)$. Although the count of all three types of lesions showed a
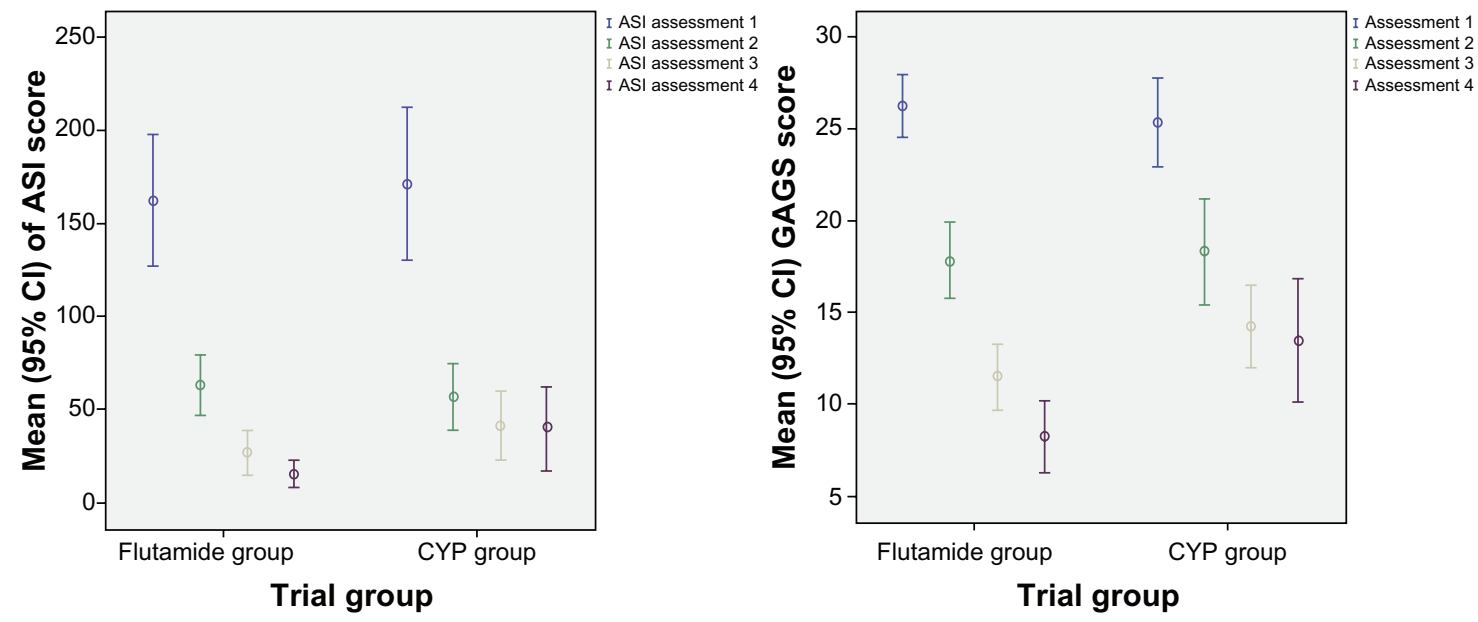

Figure 2 Error bar graph of improvement trend of ASI and GAGS score compared between flutamide and cyproterone combination. Abbreviations: ASI, Acne Severity Index; CYP, cyproterone; GAGS, Global Acne Grading System; CI, confidence interval. 
Table I Comparing the efficacy of flutamide versus cyproterone in both per protocol and intention to treat analyses

\begin{tabular}{lllllll}
\hline Risk statistic & \multicolumn{2}{l}{ Intention to treat analysis } & & \multicolumn{3}{l}{ Per protocol analysis } \\
\cline { 2 - 3 } \cline { 5 - 7 } & Value & $\mathbf{9 5 \%} \mathbf{C l}$ & $\mathbf{C S P}$ & & Value & $\mathbf{9 5 \%} \mathbf{C l}$ \\
\hline Relative risk & 1.18 & $0.89-1.6$ & 0.25 & 1.33 & $1.03-1.72$ \\
Risk difference & $13 \%$ & $-10.0-33.0$ & & $24 \%$ & $5-43$ \\
Number needed to treat & 7.7 & - & & 4.2 & $2.3-20$ \\
\hline
\end{tabular}

Abbreviations: $\mathrm{Cl}$, confidence intervals; $\mathrm{CSP}$, Chi-squared $P$ value.

steeper decrement trend for flutamide, the difference was not found to be statistically significant.

The ASI decreased significantly over time in both groups $(P<0.001)$. It decreased more in the flutamide group, but the observed difference was not found to be statistically significant $(\beta=-4.3 ; P=0.15)$. GAGS score decreased significantly over time in both groups $(P<0.001)$. The decrement trend was more prominent for the flutamide group $(\beta=-1.2$; $P<0.001)$. After 6 months of treatment, $96 \%$ of subjects in the flutamide group versus $72 \%$ in the cyproterone-estradiol combination group improved, shifting down to mild acne from their moderate acne when they were enrolled into the study $(P<0.05)$. Comparison of dichotomous primary endpoint statistics for drug efficacy in both the per protocol and intention to treat analyses are reported in Table 1 .

Satisfaction regarding different aspects of drug efficacy and tolerability was not found to be significantly different between the trial groups except for improvement in greasy skin $(P<0.05)$. Drug side effects were few, and there were no statistically significant differences between groups for tolerability. Minor scalp and face peeling, angular cheilosis, and mild gastrointestinal side effects were observed. One case of vague chest pain was also reported.

\section{Discussion}

Topical and oral antiandrogens have been recommended for use in the treatment of acne. Cyproterone acetate and newer cyclic oral contraceptives with strong antiandrogenic activity can be considered in this regard..$^{1,10,13-15}$ Flutamide is quite new in acne research. For acne associated with polycystic ovary syndrome, a combination of flutamide and the oral contraceptive pill was found to be beneficial in a randomized control of 119 women followed up for 12 months. ${ }^{16}$ In the present study, although flutamide in the per protocol analysis appeared to be more effective than the cyproterone-estradiol combination, considering the substantial number of subjects lost to follow-up and also the limited information available for flutamide in dermatology research, we had to rely more on the intention to treat analysis results, which suggest similar efficacy. However, considering all trends and descriptive statistics in this study and taking into account the small size of our trial, we conclude conservatively that flutamide is at least as effective than cyproterone for treatment of moderate acne, but this needs to be tested in larger studies. Combining flutamide with oral contraceptives may help in reducing the flutamide dose in future trials.

\section{Conclusion}

Flutamide appeared to be at least as effective as the cyproterone-estradiol combination in some aspects of acne treatment. However, this requires confirmation in a larger trial. A limitation of the study was its rather small sample size, so it must be considered as a pilot study. However, from an ethical point of view, this is usually the case for preliminary clinical trials in new drug indications. The second limitation was regarding the number of cases lost to follow-up. Although we tried to accommodate this during the statistical analysis, we need to be cautious about drawing a strong conclusion. The main strength of the study was its novelty in presenting a new treatment modality for moderate acne as well as a detailed analysis of the results using a suitable statistical methodology.

\section{Disclosure}

The authors report no conflicts of interest in this work.

\section{References}

1. Kurokawa I, Danby FW, Ju Q, et al. New developments in our understanding of acne pathogenesis and treatment. Exp Dermatol. 2009;18(10):821-832.

2. Ingram JR, Grindlay DJ, Williams HC. Management of acne vulgaris: an evidence-based update. Clin Exp Dermatol. 2010;35(4): 351-354.

3. George R, Clarke S, Thiboutot D. Hormonal therapy for acne. Semin Cutan Med Surg. 2008;27(3):188-196.

4. Haroun M. Hormonal therapy of acne. J Cutan Med Surg. 2004; 8(Suppl 4):6-10.

5. Katsambas AD, Dessinioti C. Hormonal therapy for acne: Why not as first line therapy? Facts and controversies. Clin Dermatol. 2010;28(1):17-23.

6. Thiboutot D. Acne: hormonal concepts and therapy. Clin Dermatol. 2004;22(5):419-428.

7. Adalatkhah H, Sadeghi-Bazargani H, Mini-sani N, Zeynizadeh S. Melasma and its association with different types of nevi in women: a case-control study. BMC Dermatol. 2008;8:3. 
8. Franks S, Layton A, Glasier A. Cyproterone acetate/ethinyl estradiol for acne and hirsutism: time to revise prescribing policy. Hum Reprod. 2008;23(2):231-232.

9. De Leo V, Morgante G. Different effectiveness of cyproterone acetate doses in treatment of acne. Clin Endocrinol (Oxf). 2003;58(2):246.

10. Couzinet B, Pholsena M, Young J, Schaison G. The impact of a pure anti-androgen (flutamide) on LH, FSH, androgens and clinical status in idiopathic hirsutism. Clin Endocrinol (Oxf). 1993;39(2):157-162.

11. Doshi A, Zaheer A, Stiller MJ. A comparison of current acne grading systems and proposal of a novel system. Int J Dermatol. 1997;36(6): 416-418.

12. Fitzmaurice G, Laird N, Ware J. Marginal Models: Generalized Estimating Equations. New York, NY: John Wiley and Sons; 2004.

13. Iraji F, Momeni A, Naji SM, Siadat AH. The efficacy of topical cyproterone acetate alcohol lotion versus placebo in the treatment of the mild to moderate acne vulgaris: a double blind study. Dermatol Online J. 2006;12(3):26.
14. Koltun W, Lucky AW, Thiboutot D, et al. Efficacy and safety of $3 \mathrm{mg}$ drospirenone $/ 20 \mathrm{mcg}$ ethinylestradiol oral contraceptive administered in 24/4 regimen in the treatment of acne vulgaris: a randomized, doubleblind, placebo-controlled trial. Contraception. 2008;77(4):249-256.

15. Stecova J, Mehnert W, Blaschke T, et al. Cyproterone acetate loading to lipid nanoparticles for topical acne treatment: particle characterisation and skin uptake. Pharm Res. 2007;24(5):991-1000.

16. Calaf J, Lopez E, Millet A, et al. Long-term efficacy and tolerability of flutamide combined with oral contraception in moderate to severe hirsutism: a 12-month, double-blind, parallel clinical trial. J Clin Endocrinol Metab. 2007;92(9):3446-3452.

\section{Publish your work in this journal}

Clinical, Cosmetic and Investigational Dermatology is an international, peer-reviewed, open access, online journal that focuses on the latest clinical and experimental research in all aspects of skin disease and cosmetic interventions. All areas of dermatology will be covered; contributions will be welcomed from all clinicians and basic science researchers globally. This journal is indexed on CAS. The manuscript management system is completely online and includes a very quick and fair peer-review system, which is all easy to use. Visit http://www.dovepress.com/testimonials.php to read real quotes from published authors.

Submit your manuscript here: http://www.dovepress.com/clinical-cosmetic-and-investigational-dermatology-journal 